\title{
Cognitive rehabilitation: an important tool in disability improvement after brain injuries
}

\author{
Ioana Stanescu, Gabriela Dogaru
}

\begin{abstract}
Clinical Rehabilitation Hospital Cluj-Napoca, University of Medicine and Pharmacy ClujNapoca
\end{abstract}

\begin{abstract}
Cognitive rehabilitation is as an important goal of rehabilitation therapy, which aims to help the person with neurological disability to acquire the highest level of cognitive functioning and of functional autonomy. Cognitive impairments in memory, language, judgement, attention, visuo-spatial perception are important blocks in acquiring functional independence. Cognitive rehabilitation therapy is "a systematic, functionally oriented service of therapeutic cognitive activities directed to achieve functional changes by reinforcing, strengthening or establishing previously learned patterns of behavior or establishing new patterns of cognitive activity or mechanisms to compensate ". The cognitive treatment programs include retraining strategies or the use of compensatory skills, but also counseling, environmental reorganization and use of educational and vocational training facilities. Cognitive rehabilitation requires a sustained and coordinated effort from a large team, including the patient, family and caregivers. There is an increasing evidence supporting the role and efficacy of cognitive rehabilitationin each cognitive domain. In the future, there is a need to develop innovative treatments, aiming to modulate cortical networks and to obtain long term improvements of all cognitive functions.
\end{abstract}

Key words: brain injuries, cognitive training, rehabilitation, cognitive domains neurological disability to acquire the highest level of cognitive functioning and of

Cognitive rehabilitation includes the strategies and methods used for the recovery of damaged cognitive functions (language /communication, attention, memory, calculation, visuo-spatial perception, praxis and executive functions / problem solving abilities). This therapeutic modality has an increasing development in the last years, as shown by many clinical studies. It becames more and more important to include enhancement of cognitive skills as an important goal of rehabilitation therapy, which aims to help the person with functional autonomy. Cognitive impairments in memory, language, judgement, attention, visuo-spatial perception are important blocks in acquiring functional independence. Cognitive retraining is a central part of any treatment program designed for brain-injured patients.

Cognitive, emotional and behavioural impairments are extremely common following acquired brain injuries of different etiologies, in particular traumatic brain injury (TBI) and stroke being the most frequent etiologies, but also in inflammatory 
or neurodegenerative diseases. There is a rising evidence for the effectiveness of this therapeutic modality, the first guidelines were published in 1992 [1], and the first review which include results of clinical trials was released in 1999 [2]. A Cochrane review of 6 RCTs shows a benefit of cognitive rehabilitation after stroke on attention deficits [3].

Cognition contains processing systems for various types of domain-specific informations; is a complex collection of mental skills [4] that includes various domains: attention, executive functions problem solving skills, reasoning, memory, perception, comprehension, communication, learning, visuospatial skills. After a brain injury, one or more of these skills are lost completely or partially. Cognitive rehabilitation is the science of restoring or enhancement of these processes, helping the patient to acquire the highest level of cognitive functioning possible after the injury. Progress in cognitive functioning is always associated with significant improvements in disability levels, functioning and daily life activities participation. Four cognitive domains are critical to daily life function: communication, memory, executive function / problem solving and visuo-spatial perception.

Cognitive rehabilitation therapy (CRT) definition was first established in 1997 by Bergquist as "a systematic, functionally oriented service of therapeutic cognitive activities [5] directed to achieve functional changes by reinforcing, strengthening or establishing previously learned patterns of behavior or establishing new patterns of cognitive activity or mechanisms to compensate ".

The cognitive rehabilitation program must be based on the results of physical evaluation and on cognitive and psychological assessments. Standardized neuropsychological and psychometric assessments, tests, questionnaires and interviews should be used to elaborate a complex map of patient's cognitive functions. Whenever possible, the results of a such complex evaluation should be explained to the patient or/and caregiver.

The results are used in establishing the "outcome goals" (achievements expected after participation in the training program), which should be clearly explained and agreed with the patient. The cognitive rehabilitation interventions are goaldirected. Goal setting is an essential part of the rehabilitation process and should focus directly on the impact of cognitive impairments in patient's day to day life. The acronym is SMART goals, which means that patient's objectives selected for interventions are Specific, Measurable, Achievable, Realistic and Time-limited.

However, after severe brain injuries, cognition is often inadequately or incompletely assessed, cognitive disorders being under-diagnosed and cognitive rehabilitation treatments under-prescribed.

The cognitive treatment programs include retraining strategies or the use of compensatory skills, but also counseling, environmental reorganization and use of educational and vocational training facilities. Recovery of function following brain injuries depends on restoration of lost functions or on compensatory reorganization 
of the damaged neural modules supporting particular cognitive domains [6]. Other treatment modalities used are physical therapy, family interventions, community reentry programs. Because of the complexity of these programs, a interdisciplinary team should be involved. It is essential to consider the patient as a full member of the team, participating not only in the setting of therapeutic methods, but also in progress monitoring. Rehabilitation success depends on the collaboration with the patient and with his family members.

A complete assessment of all cognitive functions should precede the onset of CRT (cognitive rehabilitation therapy). In previous years, assessment focused on disability produced by cognitive impairments, but now it is focused on the impact on the quality of life of the cognitive deficit. Usually, impairments on multiple cognitive domains appear after a brain injury, and assessment of each cognitive domain in recommended before CRT onset.

\section{Disorders}

of

communication: are common after brain injuries, especially after strokes and TBI. Communication is required for most daily activities, and important disability is related to communication disorders. Disorders of communication include aphasia, cognitivecommunication disorders, dysarthria and apraxia of speech.

Assessment of communication impairment should be performed by a speech and language therapist; consists in: interview, conversation, observation - faceto-face or by telerehabilitation and standardized tests [7]. The following items should be assessed: speech, language, cognitive-communication, reading, writing. Impact on quality of patient's life should also be specified.

Treatment of communication disorders:

- a). Treatment of aphasia is indicated in the very early phases after stroke (at 3 days) [8] or after TBI, but continues to be efficacious also in chronic phases (after 6 months) [9]. Intensive sessions of aphasia therapy are, in some opinions, more efficacious, but there is no consensus regarding the frequency, duration or distribution of séances.

Classical methods includes restorative approaches (naming objects and description of their function, conversations with the therapist, answering questions, completing missing words from sentences, selecting words to fit in a category, generating definitions, etc. ), compensatory strategies (elliptical/ incomplete speech, letter and picture boards,writing words). A different approach is constraint-induced aphasia therapy, which is based on constraining compensatory communication such as gesturing, drawing or elliptical speech, and encourages intensly verbal communication.

Therapy could be provided by a speech and language therapist or could be computer-based. Studies have shown better improvements if the patient is training with a communication partner (caregiver, family member). Group therapy appear to be better in some studies than single-subject approaches. Pharmacotherapy have been shown to add some benefits to speech and language therapy; drugs that appear beneficial are donepezil, memantine and 
galantamine. Brain stimulation techniques, such as repetitive transcranial magnetic stimulation, are still under study and could probably be beneficial in combination with speech language therapy [7].

- b). Treatment of cognitivecommunication disorders. Dysarthria refers to a speech disorder resulting form motor deficit or incoordination of the muscles involved in speech production (oral, pharyngeal, laryngeal and respiratory mmuscles). Apraxia of speech refers to a disorder of motor planning of muscular programs involved in sound production. Treatment modalities used involve behavioral treatments (methods which improves articulation, respiration, prosody, resonance) and the use of augmentative and alternative communication devices (picture boards, spelling boards, electronic devices). Gesture and writing could partially replace verbal communication. Telereabiltation techniques (distance learning programs) are recommended when speech and language therapy cannot be provided directly to the patient by a specialized person. Social participation in community-based programs could improve patient's quality of life and promote well-being.

\section{Disorders of attention.}

Attention deficits appear after many types of brain injuries, especially stroke and TBI. Attention allow our brain to focus on an important event or information while ignoring other events that are going on at the same time. Attention skills are necessary for registering an information and using it in thinking, learning, problem-solving or memory processes. Attention spam means the amount of time we can focus on one thing; attention shifting is changing the focus from one event to another, more important; divided attention is required to follow two or more processes on the same time. Rios et al. [11] divided attention in four subprocesses: cognitive flexibility, speed of processing, interference and working memory.

During the acute phase of recovery there is insufficient evidence to support the use specific attention training modalities. Therapy of attention deficits is recommended in postacute rehabilitation after stroke or TBI. Methods should include direct attention training and metacognitive training for improving development of compensatory strategies and foster generalization to real world tasks [12]. Repeated practice with a therapist ("attention process training") includes training with different stimulus modalities followed by monitoring subject's performance, providing feed-back and reinforcements and by development of strategies to compensate for attentional deficits - are used as basic treatment methods, together with computer-based interventions. Attention training seems to be more effective when directed at improving subject's performance on functional, complex tasks requiring the regulation of attention [13]. General cognitive interventions for other cognitive domains (such as memory) have also been shown to improve attention skills.

Kim at all [10] have shown that TBI patients who completed attentional training program showed activation of attentional cortical networks on functional MRI: decreased frontal lobe activity and increased 
function in the anterior cingulated and precuneus cortical areas in comparison with pre-training data.

\section{Disorders of functional}

memory are very common: $25 \%$ of patients with TBI and more than $30 \%$ of patients who have suffered a stroke have memory impairments. Memory refers to the ability to store, retain and recall information, events and procedures. According to the length of time between the exposure to stimuli and recall, memory is divided in immediate (working memory), short term (recent) and long term (remote) memory. Verbal memory refers to the ability to recall items encoded in words (heard or read). Procedural or implicit memory refers to the ability to retain how to perform an action. Explicit memory means the ability to recall voluntary an information. Attention process and executive control skills are also important in memory functioning.

Therapies for memory enhancement should reduce distraction factors from the environment and increase the efficiency of encoding and recall. The strategies used are directed towards restoring or towards compensating the lost or damaged memory function. Some training techniques are designed to improve learning and retrieval in everyday life, others are targeting specific domains such as remembering faces, routines, dates, names or appointments, and more complex techniques work on memory subgroups, such as working, episodic or prospective memory.

Compensatory strategies in memory training includes internalized strategies and external memory assistive technology. Internalized strategies consists in mnemonic techniques (e.g. diaries, visual imagery) and behavioral strategies (e.g. keeping the keys always in the same place). Self-instructional recall techniques are other internalized strategy that use self-cueing for recalling of autobiographical events and for planning actions. External assistive strategies and tools includes the use of external memory aids: diaries, pagers, PDAs, mobile phones, notebooks, computers, portable voice organizers....Both strategies are recommended for patients with mild to moderate memory impairments.

For people with severe memory impairments is recommended errorless learning technique, in which the therapist structures the learning process in order to avoid errors. Errorless technique is effective for learning specific skills of knowledge, but with limited transfer to novel tasks. The opposite technique - trial-and-error training, which favor guessing - could be used in learning $\mathrm{ADL}$ tasks. For severe impaired patients, use of externally-directed assistive devices (pagers, notebooks, computers ) appears to be beneficial.

The spaced retrieval technique use the presentation of an item in repeated trials over time, which favors learning and retaining of that information. Music therapy may be reasonable for improving attention and verbal memory, even in the early phases of rehabilitation [7].

Memory training in virtual environments could be beneficial in improving verbal and spatial knowledge.

4. Disorders of praxis and motor planning systems. Limb apraxia is defined as “ a decrease or difficulty in performing purposeful, skilled movements 
that cannot be attributed to hemiplegia" [14]. It is more common after left than after right brain injuries [7], and is associated with reduced independence in daily activities. Left parietal and supplementary motor area (SMA) are involved in producing of skilled purposive movements, which is called "praxis", and includes gesturing, pantomiming object use, programming motor sequences. In lack of praxis (apraxia), new movement programs are needed for every task [6], no matter how familiar it is. Errors appear

There has been done little research on treatments for apraxia, the literature on recovery is minimal. Specific gestural or strategy training is recommended for treating apraxia [12]. The treatment principle is to perform the gesture training in the same context in which the gestures are commonly used; training should focus on specific activities in a specific context close to the patient's normal routines [13]. Strategy training includes verbal instructions of the therapist for initiation and execution of the task. Direct training means performing the daily tasks with the therapist, anticipating and preventing errors. Gesture training uses pictured or demonstrated gestures to produce pantomimes [6].

\section{Disorders of executive}

functions. Executive functions manage the cognitive functions of the brain, being responsible for information processing assessing the awareness of own problems, prioritization of tasks, task maintenance and shifting, reasoning, problem-solving and abstract thinking, error recognition, judgement and emotion control [7]. Awareness of patient's own abilities could be increased with the aid of metacognitive strategies: exercises requiring the patient to predict task performance, anticipate difficulties, select strategies to overcome difficulties, assess the amount of assistance required to perform the task, self-monitor the results and self-evaluate their performance during various tasks [12]. Awareness intervention could be improved with verbal self-regulation during performance of an ADL task. Patients should receive training in problem-solving strategies. Self-monitoring and selfregulation are key elements in improving executive skills.

\section{Disorders of visuo-spatial} perception. Hemispatial neglect consist in a deficit in awareness and attention of one side of the space. Right visual field testing is required for diagnosis; other tests used are line bisection tests and cancellation tests. It is a common disorder after brain injury, and impairs patient's ability to participate in the rehabilitation program.

There are two treatment strategies for neglect: "bottom-up" and "top-down". "Bottom-up" strategies aim to increase attention process for the left hemispace and internal representation for this space. "Topdown” approaches teach the patient to compensate the neglect. Therapeutic methods from both categories shown to be efficient are: prism adaptation, visual scanning training, optokinetic stimulation, virtual reality training, limb activation, mental imagery, neck vibration combined with prism adaptation [7].

Non-pharmacological interventions include physical activity and environment changes. Recently, meta-analysis suggest 
that physical activity and exercise improves cognitive functions, having a protective role against cognitive decline [15]. A stimulating and enriched environment could also improve the cognitive functions, and could be used to enhance the efficiency of the cognitive rehabilitation program [16]. Enriched environments to increase engagement with cognitive activities are recommended by recent guidelines [7].

There is not enough evidence to support the role of pharmacological interventions (donepezil, rivastigmine, dextroamphetamine) in the treatment of cognitive deficits

Cognitive rehabilitation requires a sustained and coordinated effort from a large team, including the patient, his family and caregivers. There is an increasing evidence supporting the role and efficacy of cognitive rehabilitation after brain injuries. There is a need to develop innovative treatments, aiming to modulate cortical networks and to obtain long term improvements of all cognitive domains. Larger randomized control trials are needed to establish the role and importance of cognitive training of specific cognitive domains, but cognitive rehabilitation remains an essential tool in regaining patient's independence.

\section{BIBLIOGRAPHY}

1. Harley, J. P., Allen, C., Braciszewksi, T. L., Cicerone, K. D., Dahlberg, C., Evans, S., et al. (1992). Guidelines for cognitive rehabilitation. Neuro Rehabilitation, 2, 62-67.
2. Consensus conference. Rehabilitation of persons with traumatic brain injury. NIH Consensus Development Panel on Rehabilitation of Persons With Traumatic Brain Injury, JAMA. 1999 Sep 8;282(10):974-83.

3. Loetscher T, Lincoln NB. Cognitive rehabilitation for attention deficits following stroke. Cochrane Database Syst Rev. 2013;5:CD002842.

4. Parente, R, Herrmann D, Retraining cognition, 1996, Aspen, Maryland, pg 5

5. Bergquist TF, Malec JF. Psychology: Current practice and training issues in treatment of cognitive dysfunction. NeuroRehabilitation. 1997;8(1):49-56

6. Shigaki CL, Frey SH, Barrett AM. Rehabilitation of poststroke cognition. Semin Neurol. 2014;34(5):496-503

7. Winstein CJ, Stein J, Ross A, Bates B, Cherney LR, Cramer SC, Deruyter, F; Eng JJ, Fisher B, Harvey RL, Lang CE, MacKayLyons, Ottenbacher KJ, Pugh S, Reeves MJ, Richards LG, Stiers W, Zorowitz RD. Guidelines for Adult Stroke Rehabilitation and Recovery. Stroke.2016;47:000-000.

8. Godecke E, Hird K, Lalor EE, Rai T, Phillips MR. Very early poststroke aphasia therapy: a pilot randomized controlled efficacy trial. Int J Stroke. 2012;7:635-644.

9. Allen L, Mehta S, McClure JA, Teasell R. Therapeutic interventions for aphasia initiated more than six months post stroke: a review of the evidence. Top Stroke Rehabil. 2012;19:523-535. 
10. Kim YH, Yoo WK, Ko MH, Park $\mathrm{CH}$, Kim ST, Na DL. Plasticity of the attentional network after brain injury and cognitive rehabilitation. Neurorehabil Neural Repair. 2009 Jun;23(5):468-77

11. Rios M, Perianez JA, MunozCespedes JM. Attentional control and slowness of information processing after severe traumatic brain injury. Brain Inj 2004;18:25772.

12. Cicerone KD, Langenbahn DM, Braden C, Malec JF, Kalmar K, Fraas M, Felicetti T, Laatsch L, Preston Harley J, Bergquist $\mathrm{T}$, Azulay J, Cantor J, Ashman T. Evidence-based cognitive rehabilitation: updated review of the literature from 2003 through 2008. Arch Phys Med Rehabil 2011; 92: 519-528.

13. Cappa SF, Benke T, Clarke S, Rossi B, Stemmer B, van Heugten CM. Cognitive rehabilitation, in European Handbook of Neurological Management. Volume I, chapter 40, pg 545-567, $2^{\text {nd }}$ Edition, Edited by NF Gilhus, MP Barnes and M Brainin, 2011, Blackwell Publishing Ltd.

14. Bauxbaum LJ, Haaland KY, Hallett M, Wheaton L, Heilman KM, Rodriguez A, Gonzalez Rothi LJ. Treatment of limb apraxia: moving forward to improved action. Am J Phys Med Rehabil. 2008;87:149161.

15. Sofi F, Valecchi D, Bacci D, Abbate R, Gensini GF, Casini A, Macchi C. Physical activity and risk of cognitive decline: a meta-analysis of prospective studies. J Intern Med. 2011;269:107-117.

16. Janssen H, Bernhardt J, Collier JM, Sena ES, McElduff P, Attia J,
Pollack M, Howells DW, Nilsson M, Calford MB, Spratt NJ. An enriched environment improves sensorimotor function post-ischemic stroke. Neurorehabil Neural Repair. 2010;24:802-813. 